\title{
Write Characteristics of Simple Structured Single Pole Head
}

\section{Akio TAKAYAMA, Shuichi NISHIDA, Takahiro SASAZAWA, Toru SAKURAI and Koji HONDA}

\author{
Minebea Co., Ltd. R \& D Center, 1743-1 Asana, Asaba-cho, Iwata-gun, Shizuoka-ken, 437-11 Japan
}

\begin{abstract}
The write head design for a merged MR head needs to be optimized to obtain a good write performance for a perpendicular magnetic recording application. We fabricated a write-only single pole head with the simplified structure which consists of a main pole and a thin film coil without a return yoke. The write performance of the single pole head with double-layered media was evaluated by using an MR read head. The $\mathrm{O} / \mathrm{W}$ value obtained was less than $-35 \mathrm{~dB}$, and the $P W 50$ value obtained from the differentiated curve of the readback waveform was $0.20 \mu \mathrm{m}$. The small PWso from the recorded bits attributes to the sharp magnetic transitions.
\end{abstract}

Key words: perpendicular magnetic recording, single pole head, simplified structure, write head, MR head,

\section{I . INTRODUCTION}

Perpendicular magnetic recording is expected to be an important technology for high recording density. The areal density of $10 \mathrm{Gbits} / \mathrm{in}^{2}$ and beyond was obtained experimentally, using a single-pole head and a double-layered perpendicular medium[1]. The combination of an MR head and a double-layered medium is also a promising technology for future high-density recording[2]. Muraoka et.al. pointed out the $50 \%$ linear density $\left(D_{50}\right)$ of the roll-off curve measured by the MR head increased by $70 \%$ associating with the differential equalizer[3].

If an MR head is used as a read element, a write head becomes simplified structure which consists of a main pole and a thin film coil, without a return yoke. It is because the recording process of a perpendicular magnetic recording single pole head on a double-layered medium is carried out through a main pole, a recording layer and an under-layer of the medium.

It is considered that write head design should be optimized to obtain a good write performance of the merged MR head for perpendicular magnetic recording. In this study, we developed a simple structured write-only single pole head without the return yoke on the basis of analytical results from a computer simulation. The structure and write characteristics of the write-only single pole head when read out by the MR head are discussed.

\section{II . EXPERIMENTAL}

The write magnetic field amplitude of the simplified singlepole head without a return yoke(Head A) was calculated us- ing a three-dimensional finite element method, and that of the conventional single pole head(Head B)[4] was also calculated for comparison.

The head was fabricated by a thin film process with the help of an analytical simulation. Electrical performances of the head were measured by a GUZIK read/write analyzer (RWA1601), and an MR head was used as a read head at the flying height of $40 \mathrm{~nm}$.

In order to investigate the write performance of Head A, we measured saturation characteristics for this head, and also measured for Head B as a reference. The roll-off curves and isolated waveforms when read by the MR head were measured for the following three cases concerning media and write heads. Case $\mathrm{A}$; a longitudinal medium and a ring head, Case B; a perpendicular medium and a ring head, and Case $\mathrm{C}$; a perpendicular medium and a single pole head. Differentiated pulses were derived from mathematically differential operation of row waveforms for Case B and Case C.

\section{III . RESULTS AND DISCUSSION}

\section{A. Simulation}

Analytical models of Head A and Head B are shown in Fig.1(a) and (b), respectively. These heads consist of main poles, sub-yokes, thin film coils, and a return yoke(only for Head B). The main pole material chosen was a FeZrN soft magnetic film [5] with saturation magnetization(Bs) of $2.0 \mathrm{~T}$ and an initial permeability $(\mu \mathrm{i})$ of 2000 . The sub-yoke and return yoke material chosen was a NiFe film with Bs of $1.0 \mathrm{~T}$ and $\mu \mathrm{i}$ of 2000 .

Three-dimensional finite element method was used to evaluate the write magnetic fields of each type of the heads at the applied magnetomotive force(MMF) of 0.2ATo-p. The magnetic spacing between the head and the medium was $42 \mathrm{~nm}$ where flying height, protective layer thickness, and lubricant layer thickness were all included. The coercivity of the medium was assumed to be $192 \mathrm{kA} / \mathrm{m}(24000)$ ).

Fig.2 shows the write field amplitudes of Head A and Head $\mathrm{B}$ at the middle plane of the recording layer obtained from the calculation. Head running direction is aligned along the horizontal axis and $0 \mu \mathrm{m}$ point on the horizontal axis corresponds to the center of the main-pole thickness. The write field amplitude of Head A is slightly small than that of Head $\mathrm{B}$, as shown in Fig.2. However, in spite of the small magnetomotive force of $0.2 \mathrm{ATo}-\mathrm{p}$, the write field amplitude of Head A exhibited large value of $520 \mathrm{kA} / \mathrm{m}$. It is enough to over- 
write $192 \mathrm{kA} / \mathrm{m}$ (2400Oe) Hc medium because the write field amplitude of Head $A$ is 2.7 times larger than that of the coercive force of this medium. When the recording field corresponding to the medium $\mathrm{Hc}$ of $192 \mathrm{kA} / \mathrm{m}$ was obtained, the calculated value of the gradient of write field amplitude became $1020 \mathrm{kA} / \mathrm{m} \cdot \mu \mathrm{m}^{-1}$ for head A and $740 \mathrm{kA} / \mathrm{m} \cdot \mu \mathrm{m}^{-1}$ for head B, respectively. Therefore, we assume that the recording magnetic transition region of Head $\mathrm{A}$ is smaller than Head B. It was found from the above results by a computer simulation that the single pole head without the return yoke has a satisfactory write performance.

(a) Head A ; without return yoke

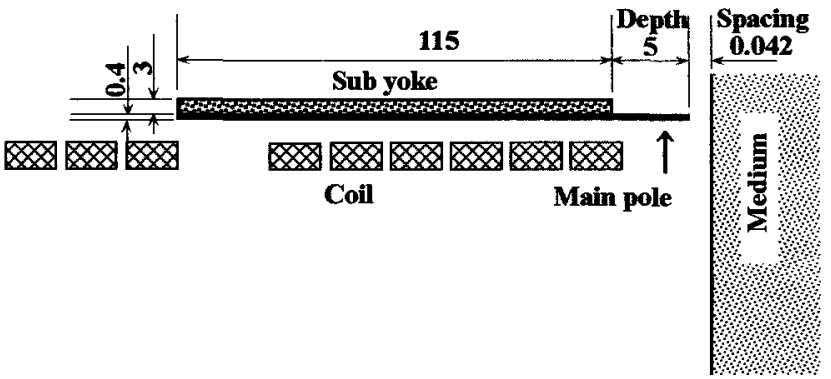

(b) Head B ; with return yoke

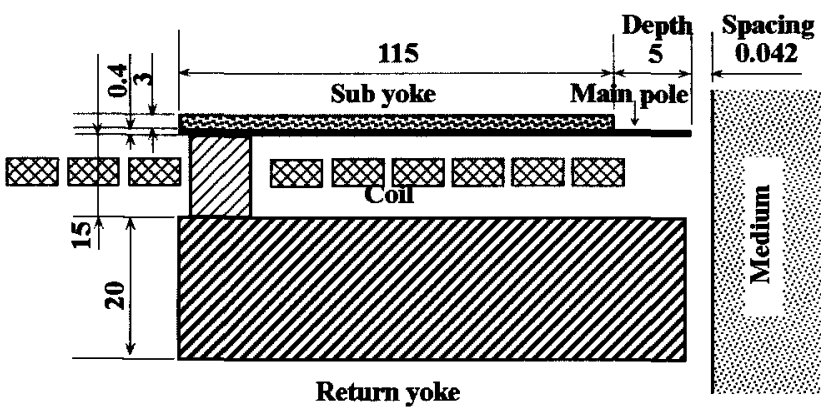

Fig.1 Analytical models of Head A and Head B.

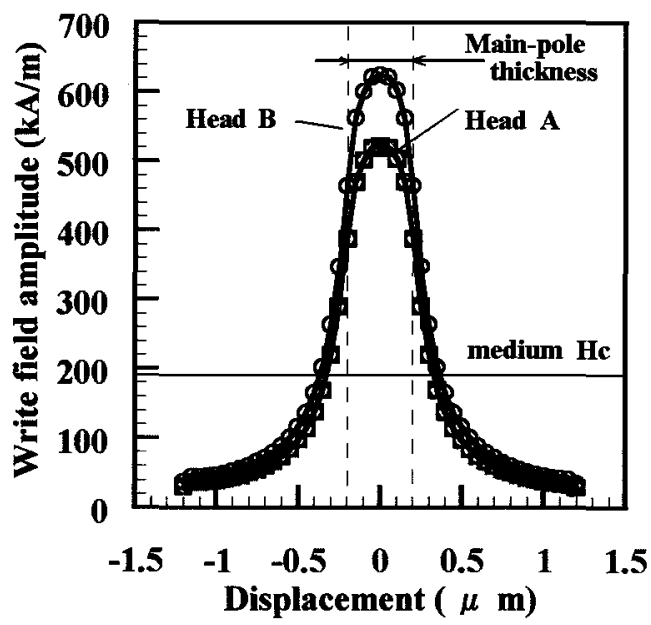

Fig.2 Write field amplitudes of Head A and Head B in the middle plane of recording layer.

\section{B. Write performances}

The cross sectional view of the simplified single pole head without the return yoke (Head A) is shown in Fig.3. The head was fabricated by a thin film process, and consisted of the main pole, the sub-yoke and the thin film coil.

In order to investigate the write performances of Head A and Head $\mathrm{B}$ (the conventinal single pole head), we examined the saturation characteristics of these heads, when an MR head with shield gap of $0.31 \mu \mathrm{m}$ was used as a read head. The saturation plots of Head A and Head B are shown in Fig.4. The low noise double layered medium with the recording layer having Bs of $0.5 \mathrm{~T}$, thickness of $50 \mathrm{~nm}$, and coercive force of $192 \mathrm{kA} / \mathrm{m}(24000 \mathrm{e})$, was used[6]. Table 1 shows experimental parameters.

The overwrite value of Head A increased more slowly than that of Head B. The cause for the poor sensitivity in the overwrite characteristics of Head A can be explained by poor head efficiency, because it has no return yoke. However, a little difference in the output amplitude and overwrite value

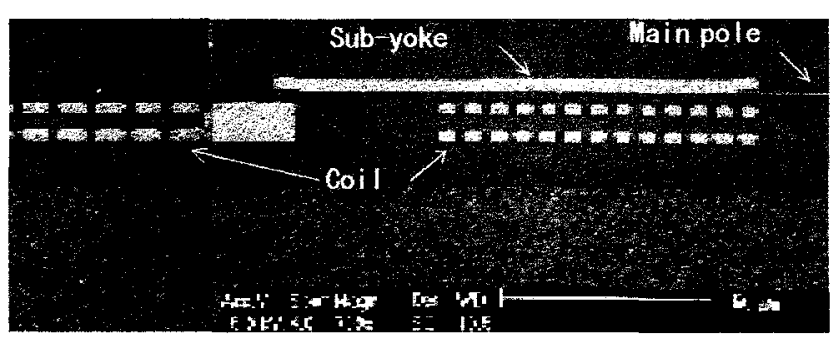

Fig. 3 Cross sectional view of simplified single pole Head A

Table 1 Experimental parameters.

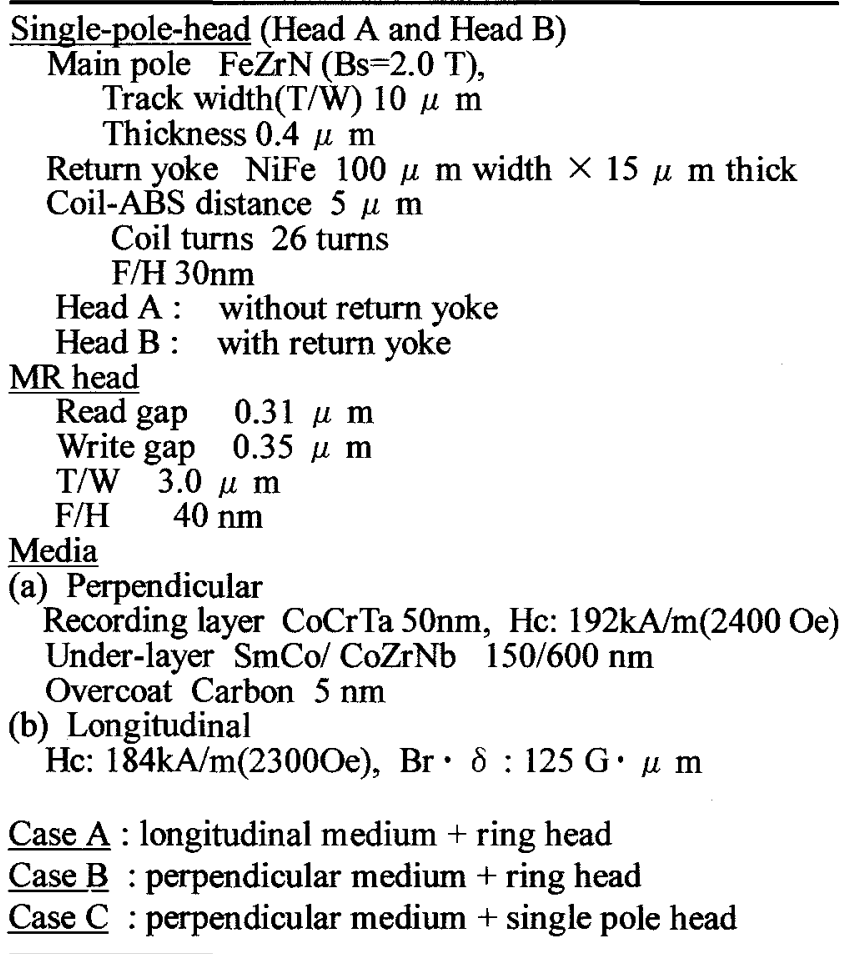


between Head A and Head B could be detected in the region of large magnetomotive force. The overwrite curve of Head A reaches the saturated value of $-35 \mathrm{~dB}$ at the magnetomotive force of 0.2 ATo-p, which should be enough for an HDD application.

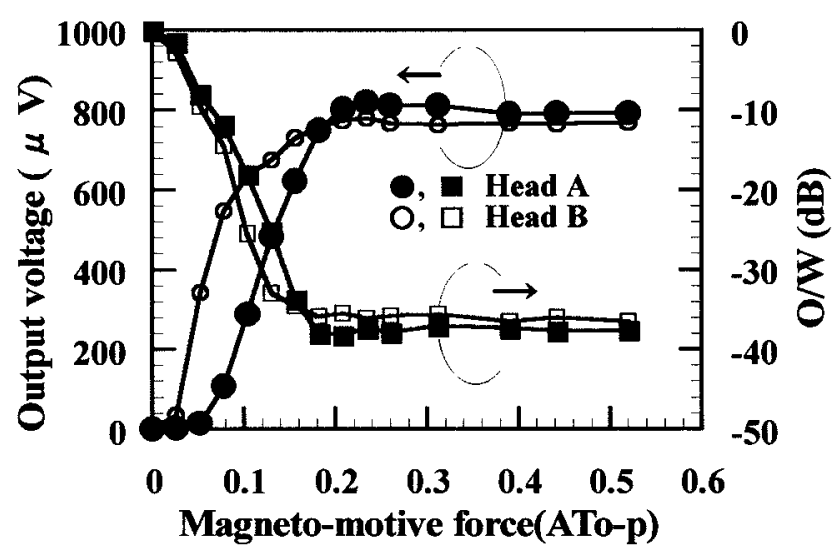

Fig.4 Saturation curves of single pole write head.

The roll-off curves of Case A, Case B, and Case C when read out by an MR head are shown in Fig.5. Head A was used in the experiment of Case $\mathrm{C}$. The longitudinal medium has $\mathrm{Br}=\delta$ of $125 \mathrm{G} \cdot \mu \mathrm{m}$ and a coercive force of $184 \mathrm{kA} / \mathrm{m}$ (2300Oe). The output amplitude of Case $\mathrm{C}$ was $20 \%$ larger than that of Case $A$ at low recording density .

D50 of the perpendicular medium(Case C) was $5.1 \mathrm{kFCmm}$ (130kFCI), which was the same as the longitudinal one(Case A). Given that $\mathrm{Br}$ is equivalent to $\mathrm{Bs}$ for perpendicular medium, due to little effect of demagnetization in high recording density region, $\mathrm{Br} \cdot \delta$ of perpendicular medium which was used in this experiment is estimated to be $250 \mathrm{G} \mu \mathrm{m}$, which is twice as much as that of the longitudinal medium. In longitudinal recording technology, utilization of a low $\mathrm{Br} \cdot \delta$ medium has realized a high density recording at the expense of output amplitude. In contrast, it seems that utilization of a high $\mathrm{Br} \cdot \delta$ medium does not hinder the high density recording for perpendicular recording within the den-

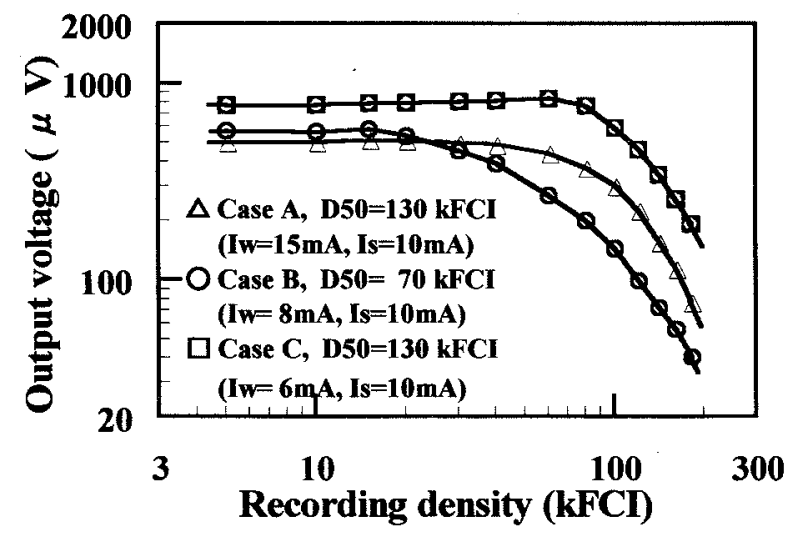

Fig.5 Roll-off curves when read by an MR head for Cases $A, B$, and $C$. sity region measured in this experiment. Dso of $5.1 \mathrm{kFCmm}(130 \mathrm{kFCI})$ was thought to be limited by the resolution of the MR head. Head B exhibited the same results as those of Head A.

On the other hand, the output amplitude of Case B showed the same value as that of Case $C$, in low recording density region. However, the amplitude rapidly decreased in high recording density region, because the sharpness of perpendicular component of the magnetic field for the ring head was likely to be inferior to that of the single pole head.

The isolated waveforms at $0.59 \mathrm{kFCmm}(15 \mathrm{kFCI})$ when read out by the MR head and their differentiated curves are shown in Fig.6, for Case A, Case B, and Case C, respectively.

The signal from the MR head for the longitudinal medium

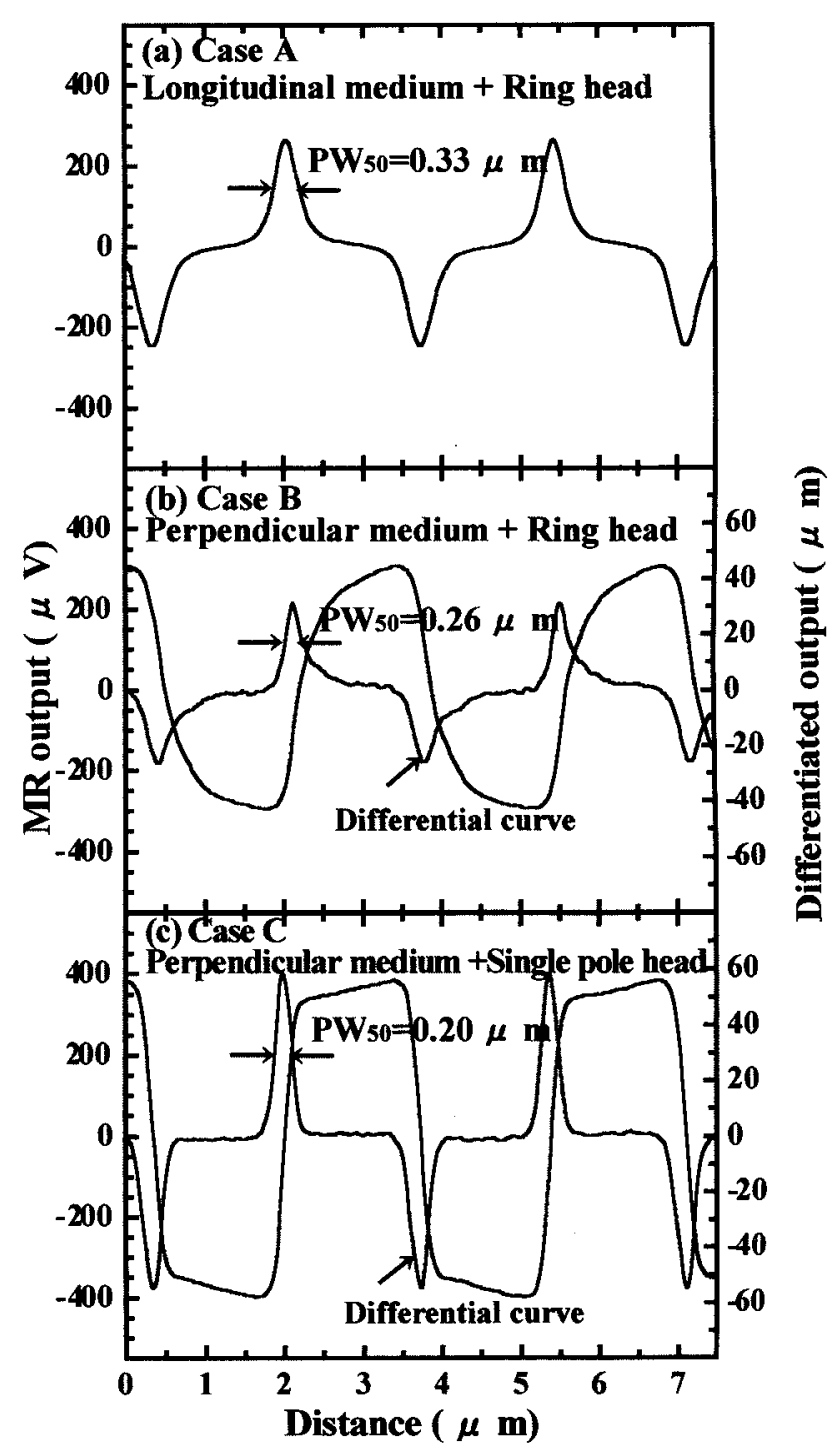

Fig.6 Isolated waveforms when read by an MR head and their differentiated curves for combination of several meadia and write head. 
shows single pulses as shown in Fig.6(a), because MR head sensitivity function has the dipulse distribution for the longitudinal medium. PW50 value obtained from isolated waveform was $0.33 \mu \mathrm{m}$.

On the other hand, the signal from the MR head for the perpendicular medium showed step-function-like wave form which is associated with the single-peak distributive sensitivity function of the read head[4]. Since differentiation of this step-function-like waveform result in single pulse waveform similar to that of the longitudinal one. The single pulse waveform shown in Fig. 6 is derived from mathematically differential operation of the measured raw waveform. PW 50 value obtained from the differentiated curve for Case $C$ was $0.20 \mu \mathrm{m}$ which is narrower than the one for the longitudinal medium, and the same results were obtained when using Head B.

We can deduce from the above results that the combination of a perpendicular double layered medium and a single-pole head shows high recording resolution, and has a possibility for the realization of high density recording, whether the singlepole head has the return yoke or not.

\section{IV . CONCLUSION}

We designed and fabricated a simple structured write-only single pole head without a return yoke for perpendicular magnetic recording.

The overwrite of this head when an MR head was used as a read head, reached the saturation value of $-35 \mathrm{~dB}$ at magnetomotive force of $0.2 \mathrm{AT}$ - $\mathrm{p}$.

The differentiated curve was derived from mathematically differential operation of the measured raw waveform. PW50 value obtained from the differentiated curve was $0.20 \mu \mathrm{m}$ which was narrower than that for the longitudinal medium. This small value suggested that recorded bits on double layered media written by this head had sharp magnetic transitions.

This head showed a good write performance and a high recording resolution, although the single pole head did not have the return yoke. Therefore, it will be important to pro- pose a new type of merged MR head combined with a simple structured single pole write head and an MR read head for perpendicular magnetic recording.

\section{ACKNOWLEDGMENT}

The authors would like to thank Mr.T.Ando and Mr.T. Nishihara of JVC for providing us with perpendicular media for this study. The authors also would like to thank Mr.M.Tomita and Dr.S.Suzuki, both of Minebea Co.,Ltd. R\&D Center, and Prof.T.Furuoya of Shizuoka Institute of Science and Technology, for their useful suggestions and supports.

\section{REFERENCES}

[1] H.Muraoka and Y.Nakamura, "Multi-Track Submicron Width Recording with a Novel Integrated Single Pole Head in Perpendicular Magnetic Recording." IEEE Trans. Magn., vol30, pp.3900-3902, Nov.1994

[2] Y.Sonobe, Y.Ikeda, H.Uchida, and T.Toyooka, "HighDensity Recording Characterization Using a Merged MR Head and Dual-Layer Perpendicular Disk." IEEE Trans. Magn., vol.31, pp.2681-2683, Nov.1995

[3] H.Muraoka, H. Yamada, Y.Nakamura, I.Abe, K.Yazawa, "Improvement of read resolution for shielded MR heads in perpendicular magnetic recording." Digest of INTERMAG97, FP-19, 1997

[4] A.Takayama, T.Sasazawa, T.Suzuki,A.Yuguchi, S.Nishida and K.Honda, "Read/Write Characteristics of a Low-Flying Single-Pole Head for Perpendicular Magnetic Recording." J.of the Magnetics Society of Japan, vol.21, pp.313-316, 1997

[5] T.Suzuki, K.Takano and Y.Nakamura, "Characterization of FeZrN Films and Their Application to Single-pole Head for PMR." J.of the Magnetics Society of Japan, vol.19(Suppiment-2), pp.38-42, 1995

[6] T.Ando and T.Nishihara, "Triple Layer Perpendicular Recording Media For High SN Ratio and Signal Stability." Digest of INTERMAG97, CP-11, 1997 